\title{
F-theory with worldvolume sectioning
}

\author{
William D. Linch III ${ }^{a}$ and Warren Siegel ${ }^{b}$ \\ ${ }^{a}$ Center for String and Particle Theory, Department of Physics, \\ University of Maryland at College Park, \\ College Park, MD 20742-4111, U.S.A. \\ ${ }^{b}$ C.N. Yang Institute for Theoretical Physics, State University of New York, \\ Stony Brook, NY 11794-3840, U.S.A. \\ E-mail: wdlinch3@gmail.com, siegel@insti.physics.sunysb.edu
}

\begin{abstract}
We describe the worldvolume for the bosonic sector of the lower-dimensional F-theory that embeds $5 \mathrm{D}, \mathrm{N}=1 \mathrm{M}$-theory and the $4 \mathrm{D}$ type II superstring. This theory is a complexification of the fundamental 5-brane theory that embeds the $4 \mathrm{D}, \mathrm{N}=1$ M-theory of the 3D type II string in a sense that we make explicit at the level of the Lagrangian and Hamiltonian formulations. We find three types of section condition: in spacetime, on the worldvolume, and one tying them together. The 5-brane theory is recovered from the new theory by a double dimensional reduction.
\end{abstract}

KeYwords: F-Theory, M-Theory, p-branes, String Duality

ArXiv EPrint: 1503.00940 


\section{Contents}

1 Introduction 1

$2 \quad 3 D$ revisited 2

3 Worldvolume action $\quad 4$

$\begin{array}{lll}4 & \text { Algebras and backgrounds } & 7\end{array}$

$\begin{array}{llr}5 & \text { Sectioning } & \mathbf{1 0}\end{array}$

5.1 Subsectioning $\mathrm{F} \rightarrow \mathrm{M} \quad 11$

$\begin{array}{lll}5.2 & \text { Subsectioning } \mathrm{F} \rightarrow \mathrm{T} & 12\end{array}$

5.3 Double dimensional reduction $4 \mathrm{D} \rightarrow 3 \mathrm{D} \quad 12$

6 Conclusions 13

\section{Introduction}

Previously [1-3] we analyzed the extension of worldsheet first-quantization of 3D string theory (S-theory), and its manifestly T-dual formulation (T-theory), to M-theory and Ftheory on branes. Our approach [3] is unrelated to any other treatment of branes in that the brane coordinates $X(\sigma)$ carry only indices that are simultaneously worldvolume and spacetime indices. They are also selfdual differential forms (similar to [4-6]). This results in section conditions involving not only spacetime (as for T-gravity [7-9] in the O(D,D) case and the analogous "F-gravity" or "exceptional geometry" for $\left.\mathrm{E}_{n(n)}[10-12]\right)$ but also the worldvolume. If extended to the full 10D type II string, this would allow for the first time an analysis of massive modes under the full STU-duality.

In this paper we describe the F-theory corresponding to type II strings in $4 \mathrm{D}$ from its formulation as a fundamental brane. This new theory is the complexification of the fundamental 5-brane theory [3] corresponding to the 3D type II string after reformulating the latter in bispinor notation. In addition to the target space sectioning constraint and Gauß's law relating target and worldvolume coordinates, the Hamiltonian analysis reveals a constraint implying a new type of sectioning quadratic in derivatives on the worldvolume. Solving these conditions reduces the F-theory to M-, T- and S-theories, as required. Alternatively, we recover the original 5-brane theory by a double dimensional reduction (corresponding to wrapping the brane on a 6-torus and then compactifying). By design, the theory's current algebra gives rise to C- and D-brackets that are covariant under the exceptional symmetry $E_{5(5)}=\operatorname{Spin}(5,5)$. After coupling to a general background, we verify explicitly that they reduce to the exceptional geometry brackets of F-gravity when truncated to massless modes. 
The remainder of this note is organized as follows: in section 2 we review the 5-brane theory corresponding to the $3 \mathrm{D}$ type II string in a formulation amenable to generalization to $4 \mathrm{D}$. This is carried out in section 3 by complexifying the coordinates in the Lagrangian formulation. The three types of constraints relating target space and worldvolume coordinates to themselves and each other are derived. They and the currents form a closed subalgebra that is studied in section 4 . In section 5 the constraints are solved, reducing $\mathrm{F}$ $\rightarrow \mathrm{M}, \mathrm{T}$, and $\mathrm{S}$. The double dimensional reduction recovering the 5-brane theory is also given. Our results are summarized in section 6 .

\section{$23 \mathrm{D}$ revisited}

Since the isotropy group $\mathrm{Sp}(4 ; \mathbf{C})$ of the F-theory for the $4 \mathrm{D}$ superstring is the complexification of that for $3 \mathrm{D}$, it will prove suggestive to review the $3 \mathrm{D}$ case here. However, the spacetime coordinates of the $4 \mathrm{D}$ case are in the spinor representation of the isometry group $\mathrm{O}(5,5)$, which begs the use of spinor notation. The result resembles the so-called "KählerDirac formalism" [13-16] in that the gauge fields, gauge parameters, etc., are represented by bispinors (polyforms).

Let us recall that the F-theory for the 3D string has a six-dimensional 5-brane worldvolume with signature $3+3$ supporting a chiral 2 -form (i.e. with self-dual 3 -form field strength) [3]. This is analogous to the fundamental string with $(1+1)$-dimensional worldsheet supporting a chiral scalar. The $3 \mathrm{D}$ string results from this description only after the dynamically-generated section conditions are imposed. (This is analogous to the reduction of the T-dual string to the usual string $[8,9]$.) We will now re-derive these constraints in a language that is easily generalized to the $4 \mathrm{D}$ string, postponing the sectioning to the $3 \mathrm{D}$ string to section 5 .

The Lagrangian for the selfdual 3-form on a 5-brane has manifest $\mathrm{O}(3,3)$ invariance [3]. In $\operatorname{Spin}(3,3)=\operatorname{SL}(4 ; \mathbf{R})$ form, the 2 -form gauge field is a real, traceless $4 \times 4$ matrix (Spin(3,3) 2-form) with 1-form gauge transformation

$$
\begin{aligned}
\delta Z_{\alpha}{ }^{\beta} & =\partial_{\alpha \gamma} \lambda^{\beta \gamma}-\partial^{\beta \gamma} \lambda_{\alpha \gamma} \\
& =2 \partial_{\alpha \gamma} \lambda^{\beta \gamma}-\frac{1}{2} \delta_{\alpha}^{\beta} \partial_{\gamma \delta} \lambda^{\gamma \delta} \quad \text { with } \quad \lambda^{T}=-\lambda .
\end{aligned}
$$

(Pairs of anti-symmetric spinor indices can be raised and lowered with $\frac{1}{2} \epsilon^{\alpha \beta \gamma \delta}$ and $\frac{1}{2} \epsilon_{\alpha \beta \gamma \delta}$.) The field strength has (anti-)selfdual parts which become symmetric bispinors

$$
F_{\alpha \beta}^{(+)}=\partial_{\gamma(\alpha} Z_{\beta)}^{\gamma} \quad \text { and } \quad F^{(-) \alpha \beta}=\partial^{\gamma(\alpha} Z_{\gamma}^{\beta)}
$$

satisfying the Bianchi identity

$$
\partial^{\alpha \gamma} F_{\beta \gamma}^{(+)}-\partial_{\beta \gamma} F^{(-) \alpha \gamma}=0
$$

This makes clear the infinite, repeating reducibility of $F \rightarrow Z \rightarrow \lambda \rightarrow \ldots$ studied in detail in reference [12]. So far, the structure is actually $\mathrm{GL}(4 ; \mathbf{R})$ covariant but invariance of the Lagrangian

$$
L=-\frac{1}{8} \operatorname{tr} F^{(+)} F^{(-)}
$$


reduces this to $\mathrm{SL}(4 ; \mathbf{R})$. (The use of $\epsilon$ changes the GL(1) weight with implications for the associated (super)gravity; cf. [2].)

Reducing to the Hamiltonian formulation requires us to break $\mathrm{SL}(4 ; \mathbf{R}) \rightarrow \operatorname{Sp}(4 ; \mathbf{R})$. Then

$$
\partial_{\alpha \beta} \rightarrow \partial_{\alpha \beta}+C_{\alpha \beta} \partial_{\tau} \quad \text { and } \quad \partial^{\alpha \beta} \rightarrow \partial^{\alpha \beta}-C^{\alpha \beta} \partial_{\tau}
$$

where now $C^{\alpha \beta} \partial_{\alpha \beta}=0$. Here $C_{\alpha \beta}$ is the $\operatorname{Sp}(4 ; \mathbf{R})$ invariant with its inverse defined by $C^{\alpha \gamma} C_{\beta \gamma}=\delta_{\beta}^{\alpha}$. (Our conventions are those of [2].) Upon lowering an index, the 2-form

$$
Z_{\alpha \beta}=\frac{1}{2}\left(X_{\alpha \beta}+Y_{\alpha \beta}\right): X_{\alpha \beta}=X_{\beta \alpha}, \quad Y_{\alpha \beta}=-Y_{\beta \alpha}, \quad C^{\alpha \beta} Y_{\alpha \beta}=0
$$

decomposes into a symmetric part $X$ (2-form) and an antisymmetric, $C$-traceless part $Y$ (vector). The field strengths reduce to

$$
\left.F_{\alpha \beta}^{(+)}=\dot{X}_{\alpha \beta}+\frac{1}{2} \partial_{\gamma(\alpha} X_{\beta}{ }^{\gamma}+\frac{1}{2} \partial_{\gamma(\alpha} Y_{\beta}{ }^{\gamma} \quad \text { and } \quad F_{\alpha \beta}^{(-)}=\dot{X}_{\alpha \beta}-\frac{1}{2} \partial_{\gamma(\alpha} X_{\beta}{ }^{\gamma}+\frac{1}{2} \partial_{\gamma(\alpha} Y_{\beta}\right)^{\gamma} \text {. }
$$

To make contact with reference [3], we now switch back to $\mathrm{SO}(2,3)$ vector notation. As mentioned, $C$-traceless anti-symmetric bispinors become vectors, symmetric tensors become 2-forms (or, equivalently, 3-forms) and the Lagrangian (2.4) becomes

$$
L=-\frac{1}{4}\left(\dot{X}_{m n}+\partial_{[m} Y_{n]}\right)^{2}+\frac{1}{12}\left(\partial_{[p} X_{m n]}\right)^{2}
$$

with all contractions performed using the $\mathrm{SO}(2,3)$ metric. The momentum conjugate to $Y$ is identically 0 whereas that conjugate to $X$ is

$$
P_{m n}=-\frac{\delta S}{\delta \dot{X}^{m n}}=\dot{X}_{m n}+\partial_{[m} Y_{n]} .
$$

The action in Hamiltonian form is expressed in manifestly $\mathrm{SO}(3,2)$-covariant notation as

$$
\begin{aligned}
S & =-\int \frac{1}{2} P_{m n} \dot{X}^{m n} d^{5} \sigma d \tau+\int H d \tau \\
H & =\int\left[\frac{1}{4} P_{m n} P^{m n}+\frac{1}{12}\left(\partial_{[p} X_{m n]}\right)^{2}+Y^{m} \partial^{n} P_{m n}\right] d^{5} \sigma .
\end{aligned}
$$

The field $Y$ remains only as a Lagrange multiplier for the Gauß law constraint

$$
\mathcal{U}_{m}:=\partial^{n} P_{m n}=0 .
$$

With the Gauß law constraint taken into account, we can gauge away the Lagrange multiplier $Y \rightarrow 0$.

The stress-energy tensor in $\mathrm{SL}(4 ; \mathbf{R})$ notation is

$$
\mathcal{T}_{\alpha \beta \gamma \delta}^{(+)}=F_{\gamma[\alpha}^{(+)} F_{\beta] \delta}^{(+)} .
$$




\begin{tabular}{|c|ccccc|}
\hline & Lagrangian & $\longrightarrow$ & Hamiltonian & $\longrightarrow$ & Current Algebra \\
\hline Symmetry & $\operatorname{Spin}(3,3)=\mathrm{SL}(4 ; \mathbf{R})$ & $\rightarrow$ & $\operatorname{Spin}(3,2)=\operatorname{Sp}(4 ; \mathbf{R})$ & $\hookrightarrow$ & $\mathrm{SL}(5 ; \mathbf{R})$ \\
Virasoro & $\mathcal{T}_{\alpha \beta \gamma \delta}^{(+)}$ & & $\mathcal{T}_{m n}, \mathcal{S}^{m}, \mathcal{T}$ & & $\mathcal{S}^{m}$ \\
Gauß & & & $\mathcal{U}_{m}$ & & $\mathcal{U}_{m}$ \\
\hline
\end{tabular}

Table 1. Symmetry breaking and enhancement in the 5-brane system (rank 4). The Lagrangian description of the dynamics preserves a larger symmetry than the Hamiltonian description. On the other hand, the Virasoro+Gauß algebra contains a kinematic subalgebra generated by $\mathcal{S}$ and $\mathcal{U}$ preserving a higher-rank exceptional symmetry.

(This form implies the symmetries $\mathcal{T}_{\alpha \beta \gamma \delta}=\mathcal{T}_{\gamma \delta \alpha \beta}=-\mathcal{T}_{\beta \alpha \gamma \delta}$ and the identity $\mathcal{T}_{\alpha[\beta \gamma \delta]}=0$.) It decomposes into $\operatorname{Sp}(4 ; \mathbf{R})=\operatorname{Spin}(3,2)$ representations $\mathcal{T}_{m n}, \mathcal{S}^{m}$, and $\mathcal{T}(\mathbf{2 0}=\mathbf{1 4} \oplus \mathbf{5} \oplus \mathbf{1})$ of which only

$$
\mathcal{S}^{r}=\frac{1}{8} \epsilon^{m n p q r} \triangleright_{m n} \triangleright_{p q} \quad \text { with current } \quad \triangleright_{m n}:=\frac{1}{4} \operatorname{tr}\left(\gamma_{m n} F^{(+)}\right)
$$

is manifestly $\mathrm{SL}(5 ; \mathbf{R})$-covariant [3]. (Recall that $F^{(+)}$was defined as a symmetric bi-spinor in $(2.7)$, so it is equivalent to a 2 -form in $\mathrm{SO}(2,3)$ that we define by the second equation.) Together, $\mathcal{S}$ and $\mathcal{U}$ form a closed subalgebra of the Virasoro algebra + Gauß law constraint with the larger-rank $E_{4(4)}=\mathrm{SL}(5 ; \mathbf{R})$ symmetry. This is summarized in table 1 .

\section{Worldvolume action}

We now give a covariant $4 \mathrm{D}$ theory by an appropriate complexification of the $3 \mathrm{D}$ case in spinor notation. The F-theory for the $4 \mathrm{D}$ string with global symmetry $E_{5(5)}=\operatorname{Spin}(5,5)$ is 16-dimensional with coordinates $X^{\mu}$ in the spinor representation. Since $X^{\mu}$ reduces in $\operatorname{Sp}(4 ; \mathbf{C})$ to a $(\mathbf{4}, \overline{\mathbf{4}})$, the $3 \mathrm{D} Z_{\alpha}{ }^{\beta}$ of $\mathrm{GL}(4 ; \mathbf{R})$ must generalize (complexify) to $Z_{\alpha}{ }^{\dot{\beta}}$ of $\mathrm{GL}(4 ; \mathbf{C})$, now lacking both trace and reality conditions.

Using $\mathrm{GL}(4 ; \mathbf{C}), \partial$ and $Z$ are both $4 \times 4$ complex matrices

$$
\partial_{\alpha \beta}, \bar{\partial}_{\dot{\alpha} \dot{\beta}}=\left(\partial_{\alpha \beta}\right)^{*}, Z_{\alpha}^{\dot{\alpha}}, \bar{Z}_{\dot{\alpha}}^{\alpha}=\left(Z_{\alpha}{ }^{\dot{\alpha}}\right)^{*}
$$

where the $\partial$ 's are anti-symmetric $(\mathbf{6}$ and $\overline{\mathbf{6}})$, and $Z\left(\mathbf{1 6}_{\mathbf{C}}\right)$ is the complexification of $X$ (which is Hermitian $X^{\dagger}=X$ ). Then the gauge transformation generalizing (2.1) is

$$
\delta Z_{\alpha}^{\dot{\alpha}}=\partial_{\alpha \beta} \lambda^{(+) \beta \dot{\alpha}}-\bar{\partial}_{\alpha \dot{\alpha} \dot{\beta}} \lambda_{\alpha}^{(-)} \quad \text { with } \quad \lambda^{( \pm) \dagger}=-\lambda^{( \pm)}
$$

anti-Hermitian gauge parameters $\left(\mathbf{1 6}\right.$ and $\left.\mathbf{1 6}^{\prime}\right)$ : $Z$ has the interpretation of a complex gauge 2-form with a complex 1-form gauge parameter. From this, we form the Hermitian matrices

$$
\begin{array}{ll}
F^{(+)}=\bar{\partial} Z+\partial \bar{Z} & \Leftrightarrow \quad F_{\dot{\alpha} \alpha}^{(+)}=\bar{\partial}_{\dot{\beta} \dot{\alpha}} Z_{\alpha}^{\dot{\beta}}+\partial_{\beta \alpha} \bar{Z}_{\dot{\alpha}}{ }^{\beta} \\
F^{(-)}=\partial Z+\bar{\partial} \bar{Z} & \Leftrightarrow \quad F^{(-) \alpha \dot{\alpha}}=\partial^{\beta \alpha} Z_{\beta}^{\dot{\alpha}}+\bar{\partial}^{\dot{\beta} \dot{\alpha}} \bar{Z}_{\dot{\beta}}{ }^{\alpha} .
\end{array}
$$


These are invariant under the gauge transformation (3.2) provided

$$
\mathcal{V}:=\frac{i}{8}\left(\partial_{\alpha \beta} \partial^{\alpha \beta}-\bar{\partial}_{\dot{\alpha} \dot{\beta}} \bar{\partial}^{\dot{\alpha} \dot{\beta}}\right)=0
$$

This is our first section condition. Assuming this, $F$ satisfies the Bianchi identity (cf. (2.3))

$$
\partial^{\alpha \beta} F_{\dot{\alpha} \beta}^{(+)}-\bar{\partial}_{\dot{\alpha} \dot{\beta}} F^{(-) \alpha \dot{\beta}}=0
$$

and its conjugate giving again the infinite, repeating reducibility $\mathbf{1 6} \oplus \mathbf{1 6}^{\prime} \rightarrow \mathbf{1 6}_{\mathrm{C}} \rightarrow \ldots$ of $F \rightarrow Z \rightarrow \lambda \rightarrow \ldots$ (cf. [12]).

As with the 5-brane, the Lagrangian

$$
L=-\frac{1}{8} \operatorname{tr} F^{(+)} F^{(-)}
$$

reduces the symmetry $\mathrm{GL}(4 ; \mathbf{C}) \rightarrow \mathrm{SL}(4 ; \mathbf{C})$. We now reduce this further $\mathrm{SL}(4 ; \mathbf{C}) \rightarrow$ $\operatorname{Sp}(4 ; \mathbf{C})$ so $\mathbf{6}=\mathbf{5} \oplus \mathbf{1}$ and $\mathbf{1 6}_{\mathbf{C}}=\mathbf{1 6} \oplus \mathbf{1 6}$ with $\overline{\mathbf{1 6}}=\mathbf{1 6}$. We define this reduction by

$$
\partial Z \rightarrow \partial Z+\frac{1}{2}\left(\dot{Z}+i Z^{\prime}\right) \quad \text { and } \quad \bar{\partial} Z \rightarrow \bar{\partial} Z+\frac{1}{2}\left(\dot{Z}-i Z^{\prime}\right)
$$

where now again $C^{\alpha \beta} \partial_{\alpha \beta}=0$ removes the trace and similarly for the conjugate $C^{\dot{\alpha} \dot{\beta}} \partial_{\dot{\alpha} \dot{\beta}}=0$. Here $C_{\alpha \beta}$ and $C_{\dot{\alpha} \dot{\beta}}$ denote the non-vanishing components of the $\operatorname{Sp}(4 ; \mathbf{C})$ invariant with the inverses defined by $C^{\alpha \gamma} C_{\beta \gamma}=\delta_{\beta}^{\alpha}$ and $C^{\dot{\alpha} \dot{\gamma}} C_{\dot{\beta} \dot{\gamma}}=\delta_{\ddot{\beta}}^{\dot{\alpha}}$. These may be used to raise and lower spinor indices. The trace, being complex, splits into two real derivatives we denote by $\dot{Z}=\partial_{\tau} Z$ and $Z^{\prime}=\partial_{\sigma} Z$. Decomposing

$$
Z=X+i Y
$$

for Hermitian $X$ and $Y$, we get the field strengths (free indices lowered)

$$
\begin{aligned}
& F^{(+)} \rightarrow[\dot{X}+i(\partial-\bar{\partial}) Y]+\left[Y^{\prime}-(\partial+\bar{\partial}) X\right] \\
& F^{(-)} \rightarrow[\dot{X}+i(\partial-\bar{\partial}) Y]-\left[Y^{\prime}-(\partial+\bar{\partial}) X\right] .
\end{aligned}
$$

The action reduces to

$$
S=-\frac{1}{2} \int\left\{\left[\dot{X}+\frac{i}{2}(\partial-\bar{\partial}) Y\right]^{2}-\left[Y^{\prime}-\frac{1}{2}(\partial+\bar{\partial}) X\right]^{2}\right\} d^{12} \sigma .
$$

The momentum conjugate to $X$ becomes

$$
P_{\alpha \dot{\alpha}}:=-\frac{\delta S}{\delta \dot{X}^{\alpha \dot{\alpha}}}=\dot{X}_{\alpha \dot{\alpha}}+\frac{i}{2}\left(\partial_{\alpha \beta} Y_{\dot{\alpha}}^{\beta}-\bar{\partial}_{\dot{\alpha} \dot{\beta}} Y_{\alpha}^{\dot{\beta}}\right)
$$

Because of the form of the field strengths, the action does not contain a $\dot{Y}^{2}$ term. Interpreting $X$ as the dynamical field, this means that in the Hamiltonian analysis of this system we should treat $\tau$ as the "time" parameter conjugate to the Hamiltonian. In this sense, $Y$ is not dynamical and we will gauge it to 0 presently. 
The $\mathcal{V}$ constraint (3.4) reduces to

$$
\mathcal{V} \rightarrow \frac{i}{8}\left(\partial_{\alpha \beta} \partial^{\alpha \beta}-\bar{\partial} \dot{\alpha \dot{\beta}} \bar{\partial}^{\dot{\alpha} \dot{\beta}}\right)-\frac{1}{8} \partial_{\tau} \partial_{\sigma} .
$$

A partial solution of this constraint is obtained by setting

$$
\text { (anything) })^{\prime}=0 \quad \text { and reducing } \quad \mathcal{V} \rightarrow \frac{i}{8}\left(\partial_{\alpha \beta} \partial^{\alpha \beta}-\bar{\partial} \dot{\alpha \dot{\beta}} \bar{\partial}^{\dot{\alpha} \dot{\beta}}\right)
$$

With this the action in Hamiltonian form becomes

$$
\begin{aligned}
& S=-\int P_{\alpha \dot{\alpha}} \dot{X}^{\alpha \dot{\alpha}} d^{10} \sigma d \tau+\int H d \tau \\
& H=\frac{1}{2} \int\left[P_{\alpha \dot{\alpha}} P^{\alpha \dot{\alpha}}+\left(\partial_{\alpha \beta} X_{\dot{\alpha}}^{\beta}-\partial_{\dot{\alpha} \dot{\beta}} X_{\alpha}^{\dot{\beta}}\right)^{2}+i Y_{\alpha}^{\dot{\alpha}}\left(\partial^{\alpha \beta} P_{\beta \dot{\alpha}}+\bar{\partial}_{\dot{\alpha} \dot{\beta}} P^{\alpha \dot{\beta}}\right)\right] d^{10} \sigma,
\end{aligned}
$$

where we have normalized the volume of the gauge-fixed $\sigma$ direction to 1 . Note that this expression for the Hamiltonian cannot be rewritten with manifest $\operatorname{Spin}(5,5)$ invariance (e.g. $P_{\alpha \dot{\alpha}} \rightarrow P_{\mu}$ is a chiral ten-dimensional spinor). We interpret the field $Y$ as a Lagrange multiplier for the constraint

$$
\mathcal{U}_{\alpha}^{\dot{\alpha}}=\frac{i}{2}\left(\partial_{\alpha \beta} P^{\beta \dot{\alpha}}+\bar{\partial}^{\dot{\alpha} \dot{\beta}} P_{\alpha \dot{\beta}}\right)
$$

generating a bosonic $\kappa$-symmetry; we use it to gauge $Y \rightarrow 0$. After this is imposed, the field strengths can be written in manifestly $\operatorname{Spin}(5,5)$-covariant form

$$
\triangleright_{\mu}:=F_{\mu}^{(+)}=P_{\mu}+\left(\gamma_{m}\right)_{\mu \nu} \partial^{m} X^{\nu} \quad \text { and } \quad \tilde{\triangleright}_{\mu}:=F_{\mu}^{(-)}=P_{\mu}-\left(\gamma_{m}\right)_{\mu \nu} \partial^{m} X^{\nu}
$$

after combining SL $(4 ; \mathbf{C})$ indices into the $16 \times 16$ Pauli matrices

$$
\left(\gamma^{m}\right)^{\mu \nu}=\left(\begin{array}{cc}
C^{\dot{\alpha} \dot{\beta}}\left(\gamma^{m}\right)^{\alpha \beta} & 0 \\
0 & C^{\alpha \beta}\left(\gamma^{m}\right)^{\dot{\alpha} \dot{\beta}}
\end{array}\right)
$$

of $\operatorname{Spin}(5,5)$.

The stress-energy tensor

$$
\mathcal{T}_{\alpha \beta \dot{\alpha} \dot{\beta}}^{(+)}=F_{\alpha[\alpha}^{(+)} F_{\dot{\beta} \beta]}^{(+)}
$$

satisfies $\mathcal{T}_{\alpha \beta \dot{\alpha} \dot{\beta}}^{(+)}=\mathcal{T}_{\alpha \beta \dot{\alpha} \dot{\beta}}^{(+) \dagger}=-\mathcal{T}_{\beta \alpha \dot{\alpha} \dot{\beta}}^{(+)}=-\mathcal{T}_{\alpha \beta \dot{\beta} \dot{\alpha}}^{(+)} . \quad$ It decomposes into $\operatorname{Sp}(4 ; \mathbf{C})$ representations $\mathcal{T}_{m n}, \mathcal{S}^{m}$, and $\mathcal{T}(\mathbf{3 6}=\mathbf{2 5} \oplus \mathbf{1 0} \oplus \mathbf{1})$ with

$$
\mathcal{T}_{\alpha \beta \dot{\alpha} \dot{\beta}}=F_{\dot{\alpha}[\alpha}^{(+)} F_{\dot{\beta} \beta]}^{(+)}-C \text {-traces, } \quad \mathcal{S}^{m}=\frac{1}{4} \triangleright \gamma^{m} \triangleright, \quad \mathcal{T}=\frac{1}{4} C^{\alpha \beta} C^{\dot{\alpha} \dot{\beta}} F_{\dot{\alpha} \alpha}^{(+)} F_{\dot{\beta} \beta}^{(+)} .
$$

Again only the $\mathcal{S}$ current can be written in manifestly $\operatorname{Spin}(5,5)$-covariant form: the subalgebra of currents $\mathcal{S}, \mathcal{U}, \mathcal{V}$ is manifestly $\operatorname{Spin}(5,5)$ covariant even thought the Hamiltonian description of the dynamics preserves only the $\operatorname{Sp}(4 ; \mathbf{C})$ subgroup. We summarize this in table 2 (cf. table 1 ). 


\begin{tabular}{|c|ccccc|}
\hline & Lagrangian & $\longrightarrow$ & Hamiltonian & $\longrightarrow$ & Current Algebra \\
\hline Symmetry & $\operatorname{Spin}(6 ; \mathbf{C})=\operatorname{SL}(4 ; \mathbf{C})$ & $\rightarrow$ & $\operatorname{Spin}(5 ; \mathbf{C})=\operatorname{Sp}(4 ; \mathbf{C})$ & $\hookrightarrow$ & Spin $(5,5)$ \\
Virasoro & $\mathcal{T}^{(+)}$ & & & & \\
& & & $\mathcal{T}_{m n}, \mathcal{S}^{m}, \mathcal{T}$ & & $\mathcal{S}^{m}$ \\
Gauß & $\mathcal{V}$ & & $\mathcal{U}_{\alpha \dot{\alpha}}$ & & $\mathcal{U}^{\mu}$ \\
Laplace & & & $\mathcal{V}, \partial_{\sigma}$ & & $\mathcal{V}$ \\
\hline
\end{tabular}

Table 2. Symmetry breaking and enhancement in the 4D system (rank 5). The Lagrangian description of the dynamics preserves a larger symmetry than the Hamiltonian description but again there is a kinematic subalgebra of the Virasoro+Gauß+Laplace algebra preserving a higherrank symmetry. (We name the $\mathcal{V}$ constraint "Laplace" since it acts as a Laplacian on functions, cf. eqs. (3.12) and (3.13).) Note that in this case the Lagrangian group $\operatorname{Spin}(6 ; \mathbf{C})$ is not a subgroup of the full symmetry group $\operatorname{Spin}(5,5)$.

\section{Algebras and backgrounds}

We now give an independent way to derive $\mathcal{S} \rightarrow \mathcal{U} \rightarrow \mathcal{V}$ that could be useful in cases in which we do not know the covariant action. (This method is simpler than finding $\mathcal{U}$ and $\mathcal{V}$ by closing the $\mathcal{S}$ current algebra.) The constraint $\mathcal{S}(3.19)$ is defined in terms of $\triangleright$. Defining the analogous $\tilde{\mathcal{S}}$ in terms of $\tilde{\triangleright}$,

$$
\mathcal{S}^{m}-\tilde{\mathcal{S}}^{m}=-i\left(\partial^{m} X^{\mu}\right) P_{\mu}+\mathcal{O}(\mathcal{U})
$$

(cf. [3]) we find the $\mathcal{U}$ constraint (3.15) in the form $(\not \partial X)^{\mu} P_{\mu}$. Similarly,

$$
\mathcal{U}=\frac{1}{2} \not \partial(\triangleright+\tilde{\nabla}) \quad \text { and } \quad \not \partial(\triangleright-\tilde{\nabla})=\mathcal{O}(\mathcal{V})
$$

we find $\mathcal{V}(3.13)$ appearing as $\partial^{m} X^{\mu} \partial_{m}$. Just as $\mathcal{S}$ generates worldvolume coordinate transformations, $\mathcal{U}$ generates residual gauge transformations. (Both generate what is left of local invariances once $\partial_{\tau}$ is thrown away.)

We next examine the current algebras. The covariant derivatives and symmetry currents $(3.16)$

$$
\triangleright_{\mu}=P_{\mu}+\left(\gamma_{m}\right)_{\mu \nu} \partial^{m} X^{\nu}, \quad \tilde{\triangleright}_{\mu}=P_{\mu}-\left(\gamma_{m}\right)_{\mu \nu} \partial^{m} X^{\nu}
$$

are bosonic, despite their resemblance to supersymmetry currents. Using the Poisson bracket

$$
\left[P_{\mu}(1), X^{\nu}(2)\right]=-i \delta_{\mu}^{\nu} \delta(1-2)
$$

for the momentum $P_{\mu}$ conjugate to $X^{\mu}$, the brackets of the currents are

$$
\begin{aligned}
& {\left[\triangleright_{\mu}(1), \triangleright_{\nu}(2)\right]=2 i\left(\gamma_{m}\right)_{\mu \nu} \partial^{m} \delta(1-2)} \\
& {\left[\triangleright_{\mu}(1), \tilde{\triangleright}_{\nu}(2)\right]=0} \\
& {\left[\tilde{\triangleright}_{\mu}(1), \tilde{\triangleright}_{\nu}(2)\right]=-2 i\left(\gamma_{m}\right)_{\mu \nu} \partial^{m} \delta(1-2) .}
\end{aligned}
$$


The pure-spinor-like constraint (3.19) [3]

$$
\mathcal{S}^{m}=\frac{1}{4}\left(\gamma^{m}\right)^{\mu \nu} \triangleright_{\mu} \triangleright_{\nu}
$$

has Poisson bracket with the current given by

$$
\left[\mathcal{S}^{m}(1), \triangleright_{\mu}(2)\right]=i\left(\gamma_{n} \gamma^{m}\right)_{\mu}{ }^{\nu} \partial^{n} \delta(1-2) \triangleright_{\nu}(1)
$$

Using

$$
\partial^{p} \delta(1-2) A(1) B(2)=\partial^{p} \delta(1-2) A B \frac{1}{2}((1)-(2))+\frac{1}{2} A \stackrel{\leftrightarrow}{\partial^{p}} B
$$

we find the algebra

$$
\left[\mathcal{S}^{m}, \mathcal{S}^{n}\right]=2 i \partial^{(m} \delta \mathcal{S}^{n)}-2 i \eta^{m n} \partial^{p} \delta(1-2) \mathcal{S}_{p}-\frac{i}{2} \delta\left[2 \partial^{[m} \mathcal{S}^{n]}+\left(\triangleright \gamma^{m n} \mathcal{U}\right)\right]
$$

similar to that of [3]. Here the $\partial \delta$ terms are evaluated at $\frac{1}{2}((1)+(2)), \quad \triangleright \gamma^{m n} \mathcal{U}=$ $\triangleright_{\nu}\left(\gamma^{m n}\right)_{\mu}^{\nu} \mathcal{U}^{\mu}$ with the bosonic $\kappa$-symmetry generator

$$
\mathcal{U}^{\mu}:=\left(\gamma_{m}\right)^{\mu \nu} \partial^{m} \triangleright_{\nu}
$$

found previously in (3.15). The existence of $\mathcal{U}$ immediately implies another constraint: $\left(\gamma_{m}\right)_{\mu \nu} \partial^{m} \mathcal{U}^{\nu}=\mathcal{V} \triangleright_{\mu}$ where

$$
\mathcal{V}:=\eta_{m n} \partial^{m} \partial^{n}
$$

Thus, we recover the condition (3.4) required by gauge invariance of the Lagrangian description.

The algebra of constraints generated by $\mathcal{S}, \mathcal{U}$, and $\mathcal{V}$ closes, and the new constraint gives rise to a third section condition, this time on the worldvolume. This new constraint implies the gauge invariance

$$
\delta X^{\mu}=\left(\gamma_{m}\right)^{\mu \nu} \partial^{m} \lambda_{\nu}
$$

and the gauge-for-gauge transformation

$$
\delta \lambda_{\mu}=\left(\gamma_{m}\right)_{\mu \nu} \partial^{m} \lambda^{\nu}
$$

Clearly, the gauge invariance is infinitely reducible.

The worldvolume derivative of a function $f=f(X)$ is given by

$$
\begin{aligned}
\partial^{m} f & =\partial^{m} X^{\mu} \partial_{\mu} f=\frac{1}{2}\left(\gamma^{m} \gamma_{n}+\gamma_{n} \gamma^{m}\right)^{\mu}{ }_{\nu} \partial^{n} X^{\nu} \partial_{\mu} f \\
& \equiv \frac{1}{2}\left(\gamma^{m} \gamma_{n}\right)^{\mu}{ }_{\nu} \partial^{n} X^{\nu} \partial_{\mu} f(\bmod \mathcal{U})=\frac{1}{4}\left(\gamma^{m}\right)^{\mu \nu}\left(\triangleright_{\nu}-\tilde{\triangleright}_{\nu}\right) \partial_{\mu} f \\
& \equiv \frac{1}{4}\left(\gamma^{m}\right)^{\mu \nu} \partial_{\mu} f \triangleright_{\nu}(\bmod \tilde{\triangleright}),
\end{aligned}
$$


in agreement with (4.1). Using this, we derive the Poisson bracket of two vector fields $V_{i}=V_{i}^{\mu} \triangleright_{\mu}$ for ${ }_{i}={ }_{1,2}$. Modulo second class constraints and sectioning this gives the C-bracket [8] (again with the $\partial \delta$ term evaluated at $\frac{1}{2}((1)+(2))$ )

$$
\left[V_{1}(1), V_{2}(2)\right]=2 i \partial^{m} \delta V_{1} \gamma_{m} V_{2}-i \delta\left[\delta_{\rho}^{\mu} \delta_{\sigma}^{\nu}-\frac{1}{4}\left(\gamma_{m}\right)_{\rho \sigma}\left(\gamma^{m}\right)^{\mu \nu}\right] V_{[1}^{\rho} \partial_{\mu} V_{2]}^{\sigma} \triangleright_{\nu}
$$

The truncation of this bracket to massless modes reproduces the "exceptional Courant bracket" of reference $[11,12]$.

We now include backgrounds by dressing the covariant derivative

$$
\triangleright_{\alpha}=e_{\alpha}^{\mu} \triangleright_{\mu}
$$

Using (4.14), we find that under worldsheet reparameterizations $\delta_{\lambda} \triangleright_{\alpha}=\left[i \int \lambda^{\mu} \triangleright_{\mu}, \triangleright_{\alpha}\right]$, the vielbein transforms according to

$$
\delta_{\lambda} e_{\alpha}^{\mu}=\lambda^{\nu} \partial_{\nu} e_{\alpha}^{\mu}-e_{\alpha}^{\nu} \partial_{\nu} \lambda^{\mu}+\frac{1}{4}\left(\gamma_{m}\right)_{\rho \sigma}\left(\gamma^{m}\right)^{\mu \nu} e_{\alpha}{ }^{\rho} \partial_{\nu} \lambda^{\sigma}
$$

in agreement with the results of $[11,12]$. The commutation relations in a general background are

$$
\left[\triangleright_{\alpha}(1), \triangleright_{\beta}(2)\right]=2 i \partial^{m} \delta(1-2) g_{\alpha \beta m} \frac{1}{2}((1)+(2))-i \delta(1-2) f_{\alpha \beta}{ }^{\gamma} \triangleright_{\gamma}
$$

where

$$
g_{\alpha \beta m}:=e_{\alpha}^{\mu}\left(\gamma_{m}\right)_{\mu \nu} e_{\beta}^{\nu} \quad \text { and } \quad f_{\alpha \beta}^{\gamma}:=c_{[\alpha \beta]}^{\gamma}+\frac{1}{2} c_{\delta[\alpha}{ }^{\varepsilon}\left(g^{\gamma \delta m} g_{\beta] \varepsilon m}\right) .
$$

Here the $g$ 's are defined by the first equation and the generalization

$$
\partial^{m} f=-\frac{1}{4} g^{\alpha \beta m} \partial_{\alpha} f \triangleright_{\beta}
$$

of (4.13), and the "anholonomy"-type coefficients (not anti-symmetric) are defined by

$$
c_{\alpha \beta}^{\gamma}:=\left(e_{\alpha} e_{\beta}{ }^{\mu}\right) e_{\mu}^{\gamma} \text {. }
$$

The Bianchi identity $\left[\left[\triangleright_{(\alpha}, \triangleright_{\beta}\right], \triangleright_{\gamma}\right]=0$ then implies the relations

$$
\begin{aligned}
f_{\gamma(\alpha}{ }^{\delta} g_{\beta) \delta m} & =2 e_{\gamma} g_{\alpha \beta m}-e_{(\alpha} g_{\beta) \gamma m} \\
\frac{1}{6} e_{[\alpha} f_{\beta \gamma}{ }^{\varepsilon} g_{\delta] \varepsilon m} & =\frac{1}{8} f_{\left[\alpha \beta^{\varepsilon}\right.} f_{\gamma \delta]}{ }^{\varphi} g_{\varepsilon \varphi m} .
\end{aligned}
$$

These relations are the direct analogs of those found for T-dual theories in [8] for the T-dual string, which we recover by dropping the worldvolume index $g_{\alpha \beta m} \rightarrow g_{\alpha \beta}$. 


\section{Sectioning}

New section conditions are obtained by replacing string coordinates with 0-modes [17]. In addition to the new section condition from Gauß's law found in reference [3], there is yet another type of section condition on the worldvolume coming from the Laplace constraint (4.10). We collect these conditions in the following table:

\begin{tabular}{|c|c|c|c|}
\hline Virasoro & $\mathcal{S}^{m}=\frac{1}{4}\left(\triangleright \gamma^{m} \triangleright\right)$ & & \\
\hline $\begin{array}{l}\text { dimensional } \\
\text { reduction }\end{array}$ & $\stackrel{\circ}{\mathcal{S}}^{m}:=\left(p \gamma^{m} P\right)$ & $\mathcal{U}^{\mu}=(\not \partial P)^{\mu}$ & \\
\hline $\begin{array}{l}\text { section } \\
\text { condition }\end{array}$ & $\mathcal{S}^{m}:=\frac{1}{2}\left(p \gamma^{m} p\right)$ & $\mathcal{U}_{0}^{\mu}:=(\not \partial p)^{\mu}$ & $\mathcal{V}:=\partial^{m} \partial_{m}$ \\
\hline
\end{tabular}

Since we now have 3 different types of section conditions ("strong constraints"), this might be a good place to review the method of their solution. The basic point is that these conditions are applied at 2 independent points in "function space": they take the generic form

$$
\partial \partial A=0 \quad \text { and } \quad(\partial A)(\partial B)=0
$$

for arbitrary functions $A$ and $B$ and with various reductions (contractions, symmetrizations, etc.) on the indices. Thus in momentum space

$$
p p^{\prime}=0
$$

where $p$ and $p^{\prime}$ may or may not be at the same point in function space. (In fact, our function space is disjoint, having momenta for both the worldvolume and spacetime: in particular, for the $\mathcal{U}$ section condition one of the momenta is in the worldvolume and the other in spacetime [3].)

So we have not only a quadratic constraint $p p=0$, but also a bilinear one $p p^{\prime}=0$. For example, for T-theory we have the universal constraint $p \cdot p^{\prime}=0$, taking the inner product with the signature of the T-symmetry group $\mathrm{O}(\mathrm{D}, \mathrm{D})$. For the quadratic constraint the most general solution is to pick a lightlike basis where the $\mathrm{O}(\mathrm{D}, \mathrm{D})$ metric is block off-diagonal, then choose a frame where $p$ has vanishing components in one of the 2 subspaces ("section") corresponding to this block decomposition. The bilinear constraint is then solved by taking $p$ in such a frame and finding that $p^{\prime}$ must be not only of the same form but in the same frame (i.e., in the same subspace). Conversely, given this $p^{\prime}$ we find that we could have chosen another $p$, but still in this same subspace. Thus although the frame is arbitrary, it is the same over all function space: all fields live on the same D-dimensional subspace of the original 2D-dimensional space. (This reduces T-theory to S-theory.)

Another example is the $\mathcal{U}_{0}$ constraint $p^{n} p_{m n}^{\prime}=0$ considered previously for the Ftheory of the $3 \mathrm{D}$ string. It is only bilinear, since $p^{m}$ is in the worldvolume while $p_{m n}^{\prime}$ is in spacetime. Because this constraint (and the whole formulation) is GL(5) covariant, we can always choose a frame where $p^{m}$ is in one particular direction, even before considering 


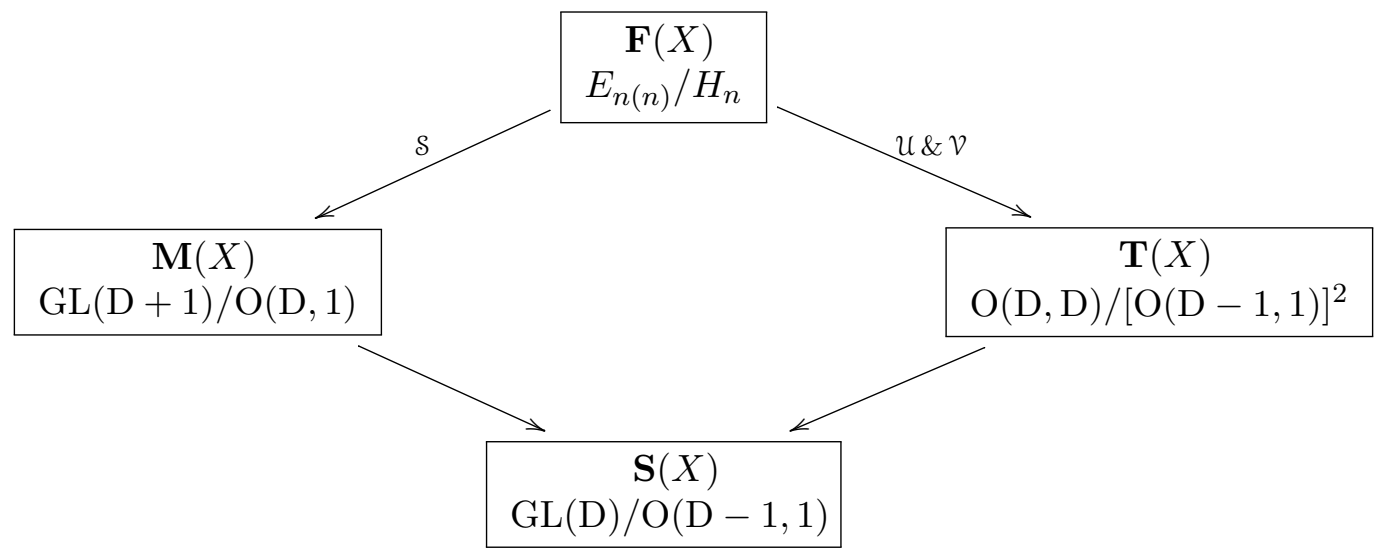

Figure 1. F-, M-, and T-theories associated to type II string (S-theory) on $X$. When the dimension of $X$ is $\mathrm{D}=3$ or 4 , there is associated to the D-dimensional type II supergravity $S(X)$ on $X$ a $(\mathrm{D}+$ 1)-dimensional $\mathrm{N}=1$ supergravity theory $M(X)$ and a D-dimensional, manifestly T-duality invariant supergravity $T(X)$. Each of these results from a theory $F(X)$ by solving the $\mathcal{S}$ constraint or $\mathcal{U}$ and $\mathcal{V}$ constraints, respectively [2].

constraints. This directly kills all of $p_{m n}^{\prime}$ carrying that index. Conversely, this general solution for $p_{m n}^{\prime}$ implies that $p^{m}$ can only point in that one direction, not only for that function, but for any function on the worldvolume. Thus again the frame is arbitrary, but applies to all functions of either the worldvolume or spacetime. (This reduces F-theory to T-theory.)

We will now carry out this reduction from $\mathrm{F}$ to $\mathrm{M}, \mathrm{T}$, and $\mathrm{S}$ for the $4 \mathrm{D}$ type II string. (See [3] for the corresponding analysis of the 3D type II string.) The solution is represented schematically in the F-theory diamond of figure 1.

\subsection{Subsectioning $\mathrm{F} \rightarrow \mathrm{M}$}

We now solve the reduction conditions (5.1b) and apply the logic above to the section constraints (5.1c). We start with $\mathcal{S}$ conditions corresponding to the reduction $\mathrm{F} \rightarrow \mathrm{M}$.

To solve the reduction and section conditions, we break $\operatorname{Spin}(5,5) \rightarrow \operatorname{GL}(5 ; \mathbf{R})=$ $\mathrm{SL}(5 ; \mathbf{R}) \times \mathrm{GL}(1 ; \mathbf{R})$. This is the same as the usual argument for $\mathrm{O}(2 n) \rightarrow \mathrm{U}(n)$ (but with split signature and real representations) so we suppress the details. Decomposing $P_{\mu} \rightarrow P^{+}, P^{m}, P_{m n}\left(\mathbf{1 6}=\mathbf{1} \oplus \mathbf{5} \oplus \mathbf{1 0}^{\prime}\right)$, and similarly for 0-modes, $\stackrel{\circ}{\mathcal{S}}$ and $\underset{\circ}{\mathcal{S}}$ become

$$
\begin{aligned}
& \stackrel{\circ}{\mathcal{S}}^{m}=\left(p \gamma^{m} P\right) \longrightarrow\left\{\begin{array}{l}
\stackrel{\circ}{\mathcal{S}^{r}}=p^{+} P^{r}+p^{r} P^{+}+\frac{1}{2} \epsilon^{m n p q r} p_{m n} P_{p q} \\
\stackrel{\circ}{\mathcal{S}}_{m}=p^{n} P_{m n}
\end{array}\right.
\end{aligned}
$$

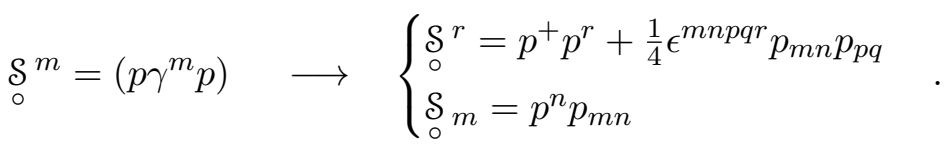


(At this point, and for the remainder of this section only, the $m, n, \ldots$ indices have become 5's.) First applying the section conditions bilinearly, we find the solution

$$
p^{+}=p^{m n}=0
$$

leaving only $p^{m}$. (Other maximal solutions correspond to a different frame for breaking to GL(5).) We then find similarly for the reduction conditions

$$
P^{+}=P^{m n}=0
$$

\subsection{Subsectioning $\mathrm{F} \rightarrow \mathrm{T}$}

Solving the $\mathcal{U}$ and $\mathcal{V}$ constraints reduces $\mathrm{F} \rightarrow \mathrm{T}$. Unlike the $3 \mathrm{D}$ case reviewed in section 2 , the existence of the $\mathcal{V}$ condition (in combination with $\mathcal{U}$ ) now restricts the one direction of the $\sigma$ derivative $\partial$ to be lightlike:

$$
\mathcal{V}=\partial^{2}=0 \quad \Rightarrow \quad \partial=\partial^{+}
$$

(The symmetry for this theory was only SO and not GL.) The $\mathcal{U}$ and $\mathcal{U}_{\circ}$ constraints thus reduce to

$$
\begin{aligned}
& \mathcal{O}=-\partial^{+} \gamma^{-} p=0 \quad \Rightarrow \quad \gamma^{-} p=0 \\
& \mathcal{U}=-\partial^{+} \gamma^{-} P=0 \quad \Rightarrow \quad \gamma^{-} P=0
\end{aligned}
$$

So we are left with a single $\sigma$ (in addition to $\tau$ ) and half (8) of the $X$ 's, i.e., a string with twice (of 4) the dimensions (T-theory).

$\mathcal{S}$ now reduces to the usual for T-theory; solving also these constraints therefore gives the $4 \mathrm{D}$ string on the worldsheet.

\subsection{Double dimensional reduction $4 \mathrm{D} \rightarrow 3 \mathrm{D}$}

Instead of solving constraints, we can perform the double dimensional reduction

$$
P^{+} \rightarrow 0, \quad P^{m} \rightarrow 0, \quad \partial_{m} \rightarrow 0
$$

(and similarly for their 0-modes) directly on the decomposition (5.4). Then it is easy to see that what remains of the constraints is precisely the reduction and section conditions of the F-theory 5-brane for the $3 \mathrm{D}$ string [3]. In other words, the F-theory for the 4D type II string contains a subalgebra of constraints defined by the worldvolume $\mathbf{5}$ and the spacetime $\mathbf{1 0}^{\prime}$ that generates the F-theory algebra for the 3D type II string. Of course it is true that the 3D type II string is embedded in the $4 \mathrm{D}$ type II string but this observation implies that the entire rank $4 \mathrm{~F}$-theory diamond (figure 1) embeds into that of rank 5 . (Reductions of only the massless part of the target space theory ("F-gravity") but for more general U-duality groups were studied in [18].) 


\begin{tabular}{|c|ccccccc|}
\hline $\mathrm{D}$ & Lagrangian $L_{n}$ & $\rightarrow$ & Hamiltonian $H_{n}$ & $\rightarrow$ & Currents $E_{n(n)}$ & $\sigma$ & $X$ \\
\hline 3 & Spin $(3,3)=\mathrm{SL}(4 ; \mathbf{R})$ & & Spin $(3,2)=\mathrm{Sp}(4 ; \mathbf{R})$ & & $\mathrm{SL}(5 ; \mathbf{R})$ & $\mathbf{5}^{\prime}$ & $\mathbf{1 0}$ \\
& $\mathrm{Spin}(6 ; \mathbf{C})=\mathrm{SL}(4 ; \mathbf{C})$ & & $\mathrm{Spin}(5 ; \mathbf{C})=\mathrm{Sp}(4 ; \mathbf{C})$ & & $\mathrm{Spin}(5,5)$ & $\mathbf{1 0}$ & $\mathbf{1 6}$ \\
\hline
\end{tabular}

Table 3. Symmetry groups of fundamental F-theory branes. The symmetry manifested by the Lagrangian and Hamiltonian formulations of the fundamental branes of F-theory corresponding to type II strings in $\mathrm{D}=3$ and 4 dimensions. The rank of the global exceptional symmetry $n=\mathrm{D}+1$ and the $E_{n(n)}$ representations of the worldsheet $(\sigma)$ and target $(X)$ coordinates are given in the last two columns.

\section{Conclusions}

In this paper we described the fundamental theory giving rise to the F-theory embedding the four-dimensional type II string (corresponding to the split form of the rank 5 exceptional group $\left.E_{5(5)}=\operatorname{Spin}(5,5)\right)$ as a complexification of that of the fundamental 5-brane of the 3D string [3]. This description requires three types of section condition: in addition to the original section condition $(\mathcal{S})$ on the target space $[11,12]$ and another $(\mathcal{U})$ relating target space to worldvolume [3], there is now also a third section condition $(\mathcal{V})$ on the worldvolume itself. The analysis of these constraints shows that the 3- and 4-dimensional theories are related by double dimensional reduction (5.10).

The structure of these theories is such that the Lagrangian theory is invariant under an a priori unknown symmetry group $L_{n}$ that is broken to the subgroup $H_{n}$ in the Hamiltonian description. This subgroup is also the (split form of the) maximal compact subgroup of the split form $E_{n(n)}$. We represent this in table 3. The algebra (4.4) of the currents is manifestly $E_{n(n)}$-covariant as is the "kinetic" subalgebra of the full Virasoro+Gauß+Laplace algebra of constraints that is generated by $\mathcal{S}, \mathcal{U}$, and $\mathcal{V}$ (eqs. (4.5), (4.9), and (4.10), resp. and cf. table 2).

Clearly, it is of interest to extend this analysis to higher rank. The next classical superstring in the series is the $6 \mathrm{D}$ type II string corresponding to the maximal global symmetry $E_{7(7)}$. In this case the naïve dimension of the worldvolume exceeds that of the target so we expect the new worldvolume section condition (and possibly new constraints) to play a role in cutting this dimension down. Since these cases correspond to superstrings, supersymmetrization of our brane systems may give insight into the fundamental theories corresponding to these higher-dimensional F-theories.

\section{Acknowledgments}

WDL3 is partially supported by the UMCP Center for String \& Particle Theory and National Science Foundation grants PHY-0652983, and PHY-0354401. Ws is supported in part by National Science Foundation grant PHY-1316617.

Open Access. This article is distributed under the terms of the Creative Commons Attribution License (CC-BY 4.0), which permits any use, distribution and reproduction in any medium, provided the original author(s) and source are credited. 


\section{References}

[1] M. Poláček and W. Siegel, T-duality off shell in 3D Type II superspace, JHEP 06 (2014) 107 [arXiv: 1403.6904] [INSPIRE].

[2] W.D. Linch III and W. Siegel, F-theory Superspace, arXiv:1501.02761 [INSPIRE].

[3] W.D. Linch III and W. Siegel, F-theory from Fundamental Five-branes, JHEP 02 (2021) 047 [arXiv: 1502.00510] [INSPIRE].

[4] D. Kutasov and E.J. Martinec, M-branes and $N=2$ strings, Class. Quant. Grav. 14 (1997) 2483 [hep-th/9612102] [INSPIRE].

[5] D. Kutasov, E.J. Martinec and M. O'Loughlin, Vacua of M-theory and $N=2$ strings, Nucl. Phys. B 477 (1996) 675 [hep-th/9603116] [InSPIRE].

[6] D. Kutasov and E.J. Martinec, New principles for string/membrane unification, Nucl. Phys. B 477 (1996) 652 [hep-th/9602049] [INSPIRE].

[7] W. Siegel, Two vierbein formalism for string inspired axionic gravity, Phys. Rev. D 47 (1993) 5453 [hep-th/9302036] [INSPIRE].

[8] W. Siegel, Superspace duality in low-energy superstrings, Phys. Rev. D 48 (1993) 2826 [hep-th/9305073] [INSPIRE].

[9] W. Siegel, Manifest duality in low-energy superstrings, in International Conference on Strings 93, (1993) [hep-th/9308133] [INSPIRE].

[10] D.S. Berman, H. Godazgar, M. Godazgar and M.J. Perry, The Local symmetries of M-theory and their formulation in generalised geometry, JHEP 01 (2012) 012 [arXiv:1110.3930] [INSPIRE].

[11] A. Coimbra, C. Strickland-Constable and D. Waldram, $E_{d(d)} \times \mathbb{R}^{+}$generalised geometry, connections and M-theory, JHEP 02 (2014) 054 [arXiv:1112.3989] [INSPIRE].

[12] D.S. Berman, M. Cederwall, A. Kleinschmidt and D.C. Thompson, The gauge structure of generalised diffeomorphisms, JHEP 01 (2013) 064 [arXiv:1208.5884] [INSPIRE].

[13] D. Iwanenko and L. Landau, Zur theorie des magnetischen elektrons, I, Z. Phys. 48 (1928) 340.

[14] C. Lanczos, Die tensoranalytischen Beziehungen der Diracschen Gleichung, Z. Phys. 57 (1929) 447 [physics/0508002] [INSPIRE].

[15] A.W. Conway, Quaternion treatment of the relativistic wave equation, Proc. Roy. Soc. Lond. A 162 (1937) 145 [INSPIRE].

[16] E. Kähler, Der innere Differentialkalkül, Rend. Mat. 21 (1962) 425.

[17] T. Kugo and B. Zwiebach, Target space duality as a symmetry of string field theory, Prog. Theor. Phys. 87 (1992) 801 [hep-th/9201040] [InSPIRE].

[18] D.S. Berman and R. Otsuki, Reductions of Exceptional Field Theories, JHEP 03 (2020) 066 [arXiv: 1911.06150] [INSPIRE]. 